Case Report

\title{
Presumed Retinal Metastasis from Lung Adenocarcinoma: A Case Report and Literature Review
}

\author{
Gökçen Özcan $\mathbb{D}^{\mathrm{D}},{ }^{1}$ Ahmet Kaan Gündüz $\mathbb{D}^{1},{ }^{1}$ Ibadulla Mirzayev $\mathbb{D}^{\mathrm{D}},{ }^{1}$ and Ayhan Sağlik $\mathbb{D}^{2}$ \\ ${ }^{1}$ Ankara University Faculty of Medicine, Ophthalmology Department, Ankara, Turkey \\ ${ }^{2}$ Harran University Faculty of Medicine, Ophthalmology Department, Şanliurfa, Turkey
}

Correspondence should be addressed to Ahmet Kaan Gündüz; drkaangunduz@gmail.com

Received 8 December 2020; Revised 3 March 2021; Accepted 10 May 2021; Published 10 June 2021

Academic Editor: Maurizio Battaglia Parodi

Copyright (C) 2021 Gökçen Özcan et al. This is an open access article distributed under the Creative Commons Attribution License, which permits unrestricted use, distribution, and reproduction in any medium, provided the original work is properly cited.

A 63-year-old Caucasian man with metastatic lung adenocarcinoma undergoing chemotherapy and external radiotherapy was referred for routine eye examination. Although he was asymptomatic, ocular examination revealed a relatively wellcircumscribed whitish retinal lesion measuring $0.5 \times 0.5 \times 0.5 \mathrm{~mm}$ located along the inferotemporal vascular arcade in the right eye. Optical coherence tomography (OCT) showed a hyperreflective dome-shaped lesion occupying the inner retinal layers with few hyperreflective dots overlying the retina in the posterior vitreous consistent with tumor cells. Fluorescein angiography revealed early hyperfluorescence and late staining without leakage at the lesion site. A diagnosis of presumed retinal metastasis from lung adenocarcinoma was made. At 2 months follow-up after completion of chemotherapy, the retinal lesion was found to have regressed completely leaving minor irregularities in the inner retinal layers on OCT. To date, there have been only 41 cases of carcinoma metastasis to the retina reported in the literature including the current case. Despite its rarity, retinal metastasis should be considered in the differential diagnosis of a white-yellow retinal mass with/without overlying vitreous cells especially in patients with a history of systemic cancer.

\section{Introduction}

Metastatic tumors of the uveal tract are the most common intraocular malignancies, whereas retinal metastases are rare compared to uveal metastases [1]. The choroid is the most common site of uveal metastasis. The choroid receives $85 \%$ of the ocular blood flow compared to the retina which receives only $5 \%$, thereby explaining the rarity of retinal metastasis [2]. Carcinomas metastasize to the choroid more often compared to sarcomas and melanomas. However, melanomas tend to metastasize to the retina more frequently than carcinomas [3]. Tumor cells are periodically shed to the circulation from the primary site. Expression of specific adhesion molecules on extravasated metastatic cells allows the proliferation of these cells on the metastatic host sites [4]. Several previously published papers reviewed vitreous and retinal metastasis from cutaneous melanomas $[5,6]$. We hereby present a case of retinal metastasis from lung carcinoma and review the literature on carcinoma metastasis to the retina.

\section{Case Report}

A 63-year-old Caucasian man diagnosed with metastatic lung adenocarcinoma 5 months ago and receiving systemic chemotherapy and external radiotherapy to the right hip underwent routine eye examination. He had no history of known ocular disease and intervention and was asymptomatic. Best corrected visual acuities were 20/32 in the right eye and 20/20 in the left eye. Intraocular pressures were $15 \mathrm{mmHg}$ and $17 \mathrm{mmHg}$ in the right and left eyes, respectively. Anterior segment examination showed both eyes had moderate nuclear sclerosis. Fundus examination of the right eye revealed a relatively well-circumscribed whitish retinal lesion measuring $0.5 \times 0.5 \times 0.5 \mathrm{~mm}$ located along the inferotemporal vascular arcade. There was no apparent vitreous and choroidal involvement. The left eye had normal funduscopic findings with blunted foveal reflex. Spectral domain optical coherence tomography (SD-OCT) of the right eye showed a hyperreflective dome-shaped lesion occupying the inner retinal layers with few hyperreflective dots overlying 


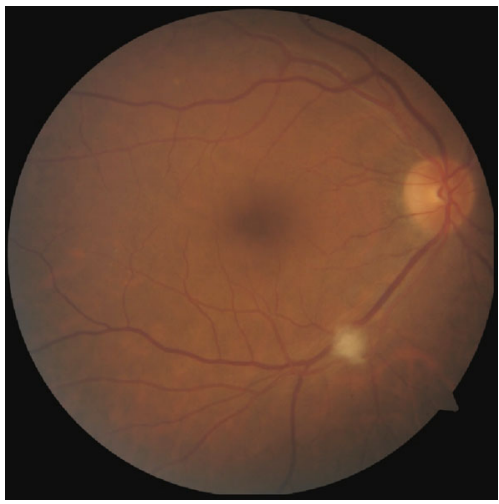

(a)

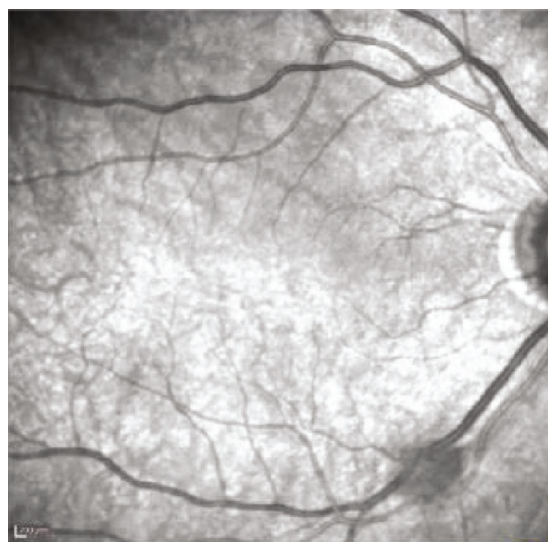

(b)

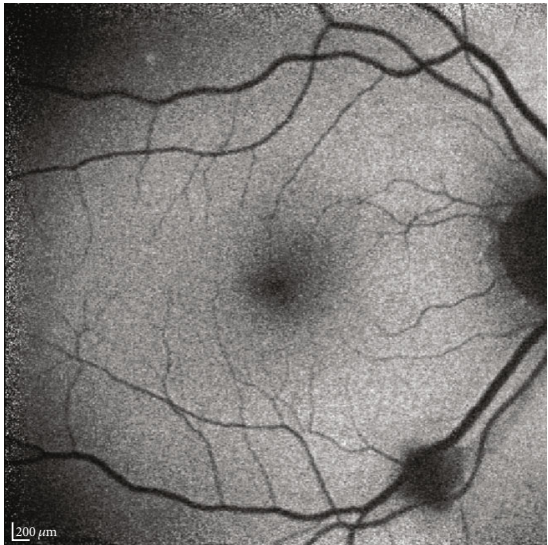

(c)

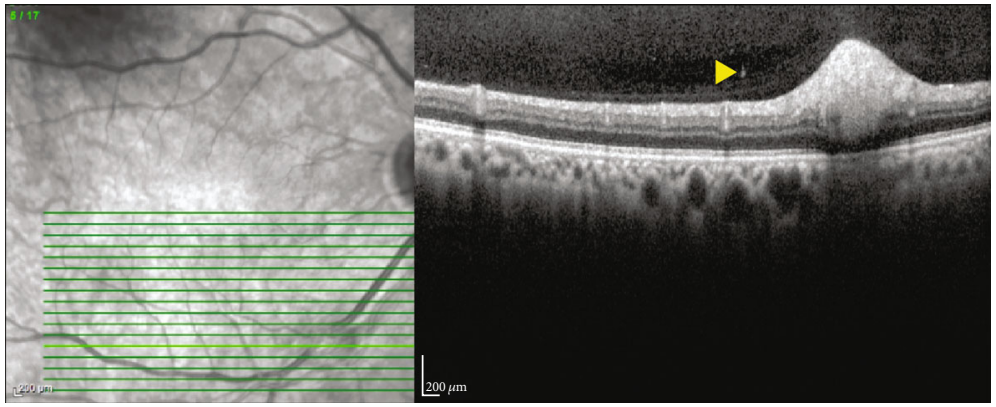

(d)

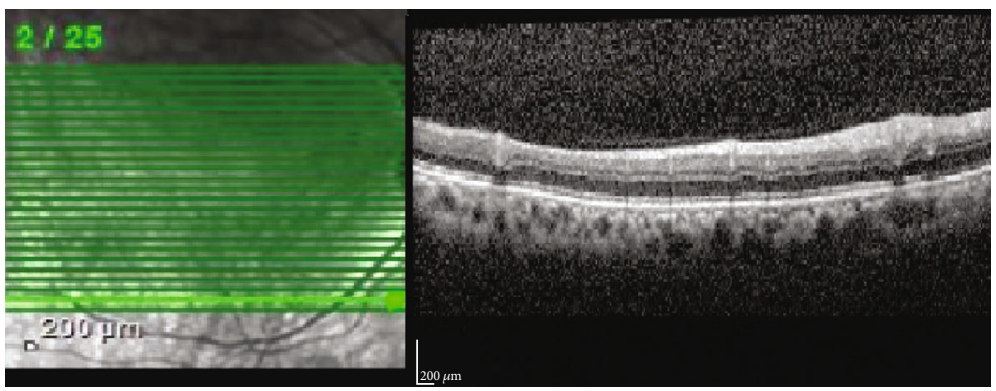

(e)

FIGURE 1: (a) Whitish perivascular retinal lesion measuring approximately 0.5 x $0.5 \mathrm{x} 0.5 \mathrm{~mm}$ located along the inferotemporal arcade in the right eye. (b) The lesion is hyporeflective on infrared imaging. (c) The lesion shows hypoautofluorescence on fundus autofluorescence imaging. (d) Spectral domain optical coherence tomography (SD-OCT) imaging shows the hyperreflective dome-shaped lesion replacing inner retinal structures, causing disorganization and compression of the outer retina. Multiple hyperreflective dots are observed overlying the retina consistent with tumor cells in the posterior vitreous (arrowhead). Ellipsoid zone cannot be observed clearly because of partial posterior shadowing by the tumor, but the retinal pigment epithelium is intact. (e) At 2 months follow-up examination after completion of chemotherapy, the retinal lesion regressed completely leaving only focal irregularities in the inner retinal layers on SD-OCT.

the retina consistent with tumor cells in the posterior vitreous. The outer retina was compressed by tumor and partial choroidal shadowing was observed without clinical or imaging evidence for choroidal involvement. The lesion was hypofluorescent on fundus autofluorescence (FAF) imaging. Fluorescein angiography (FA) revealed early hyperfluorescence of the lesion, late staining, and no leakage. The tumor was flat and nonmeasurable on B-mode ultrasonography. Brain magnetic resonance imaging revealed normal findings. Based on the clinical and imaging features, a diagnosis of presumed lung carcinoma metastasis to the retina was made. At a follow-up examination 2 months later after completion of systemic chemotherapy, the visual acuity in the right eye was 20/60 due to the worsening of the cataract. Fundus examination revealed that the retinal lesion in the right eye had regressed completely. There were only focal irregularities in the inner retinal layers on SD-OCT (Figure 1). The patient passed away 6 months after the diagnosis of retinal metastasis.

\section{Discussion}

Cutaneous melanoma is the most common metastatic retinal tumor. Tang et al. reviewed 42 cases of tumor metastasis to the retina published in the English literature from 1935 to 2017 and noted that the most common primary sites for 
metastatic tumors to the retina were cutaneous melanoma followed by lung carcinoma and gastrointestinal carcinoma [7]. Ramaesh et al. reported 39 melanoma metastases to the vitreous and retina in his literature review in 1998 [6]. Our search of the literature showed that there were at least 60 cases of melanoma metastasis to the vitreous and retina reported so far and the total number of reported carcinoma metastasis to the retina was 40 . Of the 41 cases with carcinoma metastasis to the retina including our case, 15 (36.6\%) cases had lung carcinoma, $13(31.7 \%)$ cases had gastrointestinal carcinoma including 1 case of pancreatic carcinoma and 1 case of hepatocholangiocarcinoma, 6 (14.6\%) had breast carcinoma, 3 (7.3\%) had adenocarcinoma with unknown primary site, 2 (4.9\%) had genitourinary tract carcinoma, 1 (2.4\%) had uterine carcinoma, and 1 (2.4\%) had nasopharyngeal carcinoma. The mean age of all reported cases was 56.1 (range 15-75, $\mathrm{SD} \pm 11.9)$ years. Twenty-five out of $41(61.0 \%)$ patients were males, and the remaining 16 (39.0\%) were females.

Retinal metastases are usually unilateral and solitary; however, bilateral and multifocal involvement have also been reported [8-10]. At the time of diagnosis of retinal metastasis, the majority of patients have metastasis at other sites as well (Table 1). Alternatively, ocular involvement may be the first finding of metastatic disease, and these patients must be carefully evaluated for the presence of systemic metastases at other sites.

Decreased vision, floaters, and ocular pain are the common presenting features in carcinoma metastasis to the retina. However, retinal metastasis can be asymptomatic as in our case. This underlines the necessity for patients with metastatic systemic cancer to have periodic eye examinations. Carcinoma metastasis to the retina most commonly presents as a focal, yellow-white, intraretinal mass with ill-defined borders. However, the tumor borders can sometimes be relatively well-circumscribed, as in our case. Overlying vitreous cells can be observed mimicking uveitis. Vitreous involvement may be seen as white-yellow cellular clumps or strands, sometimes hindering the view of the retina. Sometimes anterior chamber cells and a pseudohypopyon formed by tumor cells can also be observed $[9,10]$. Glaucoma secondary to the obstruction of aqueous outflow by tumor cells in the trabecular meshwork may be observed.

Metastatic retinal melanoma usually presents with a brown lesion with ill-defined borders, but amelanotic melanoma metastasis with yellow color has also been described. Vitreous metastasis from cutaneous melanoma is characterized by pigmented cellular clumps. On the other hand, retinal carcinoma metastasis is characterized by ill-defined whitish retinal infiltration with overlying nonpigmented cellular aggregates [11].

In our literature review of metastatic retinal cancer (Table 1), vitreous involvement in addition to retinal metastasis was found in 17 (41.5\%) cases. Optic disc and choroidal involvement together with retinal metastasis were reported in $5(12.2 \%)$ cases $[8,10,12-15]$. Retinal involvement usually starts from the retinal nerve fiber and ganglion cell layers. The superficial vascular plexus located in the ganglion cell layer and supplied by the central retinal artery is the major source of metastatic tumors to the retina. As retinal metasta- sis grows, it replaces deeper retinal tissue. After full-thickness retinal involvement occurs, tumor cells may break through the retina pigment epithelium/Bruchs membrane and invade the choroid $[10,16]$. Tumor cells may also penetrate the inner limiting membrane and spread into the vitreous cavity. Isolated carcinoma metastases to the vitreous have also been reported; however, histopathologic findings of enucleated eyes with isolated carcinoma metastases to the vitreous showed microscopic retinal involvement $[5,17]$.

Retinal metastases tend to be angiocentric and grow around the vessels as they are bloodborne [14, 18]. Histopathologic examination shows tumor emboli in the lumens of retinal vessels in the ganglion cell layer around retinal infiltrations $[5,14,17,19]$. Retinal vascular occlusion has been reported leading to retinal nonperfusion and neovascularization $[10,20]$. Complete disappearance of retinal metastatic lesion after chemotherapy as in our case is rare and has been reported only once before in a 15 -year-old boy with nasopharyngeal carcinoma [21].

Diagnostic methods used in retinal metastatic tumors include OCT, OCT angiography, ultrasonography, FAF, FA, fine needle aspiration biopsy, pars plana vitrectomy, and chorioretinal biopsy. The differential diagnosis of carcinoma metastasis to the retina includes cotton wool spots, retinal astrocytic hamartoma, inflammatory retinitis, and primary vitreoretinal lymphoma. The former 2 lesions originate from the retinal nerve fiber layer, whereas carcinoma metastasis to the retina demonstrates multilayer involvement of the retina. Nonpigmented vitreous cells also pose a diagnostic challenge as they can resemble vitreoretinal lymphoma, amyloidosis, candida endophthalmitis, or intermediate uveitis. Cellular aggregates in the vitreous appearing as spherules or strands should always alert the clinicians to the possibility of metastatic cells.

Treatment options for carcinoma metastasis to the retina include observation, systemic chemotherapy, intravitreal chemotherapy, plaque radiotherapy, external radiotherapy, photodynamic therapy, surgical excision, and enucleation for a blind, painful eye [22-24]. The survival rate for patients with carcinoma metastasis to the retina is poor, and the mean survival for all reported cases with retinal metastasis was only 5.7 (range $1-23, \mathrm{SD} \pm 5.2$ ) months (Table 1).

In summary, carcinoma metastasis to the retina is rare with only 41 cases being reported so far including the current case. The lung is the most common primary site for carcinoma metastasis to the retina followed by the gastrointestinal system. Retinal metastases are angiocentric, originate from the superficial vascular plexus, and are usually initially located in the inner retina. Vitreous involvement may also be seen. Patients generally have widespread metastasis at the time of diagnosis, and the life expectancy is poor. Treatment options are varied and should be tailored to the patients' needs.

\section{Method of Literature Search}

The literature search consisted of a systematic review of the literature using PubMed database till January 2021 using the following key words: "retinal metastasis" or "carcinoma 


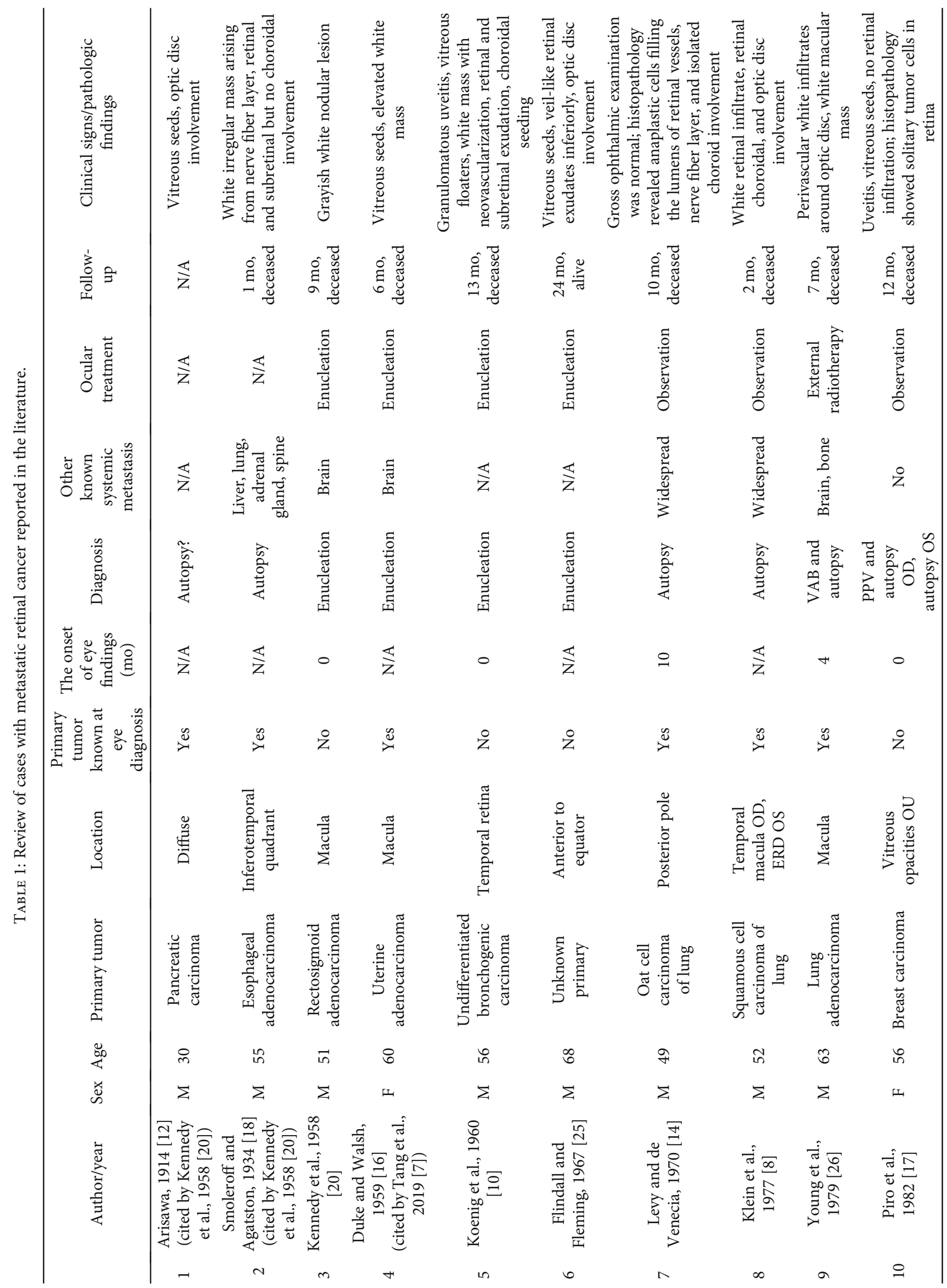




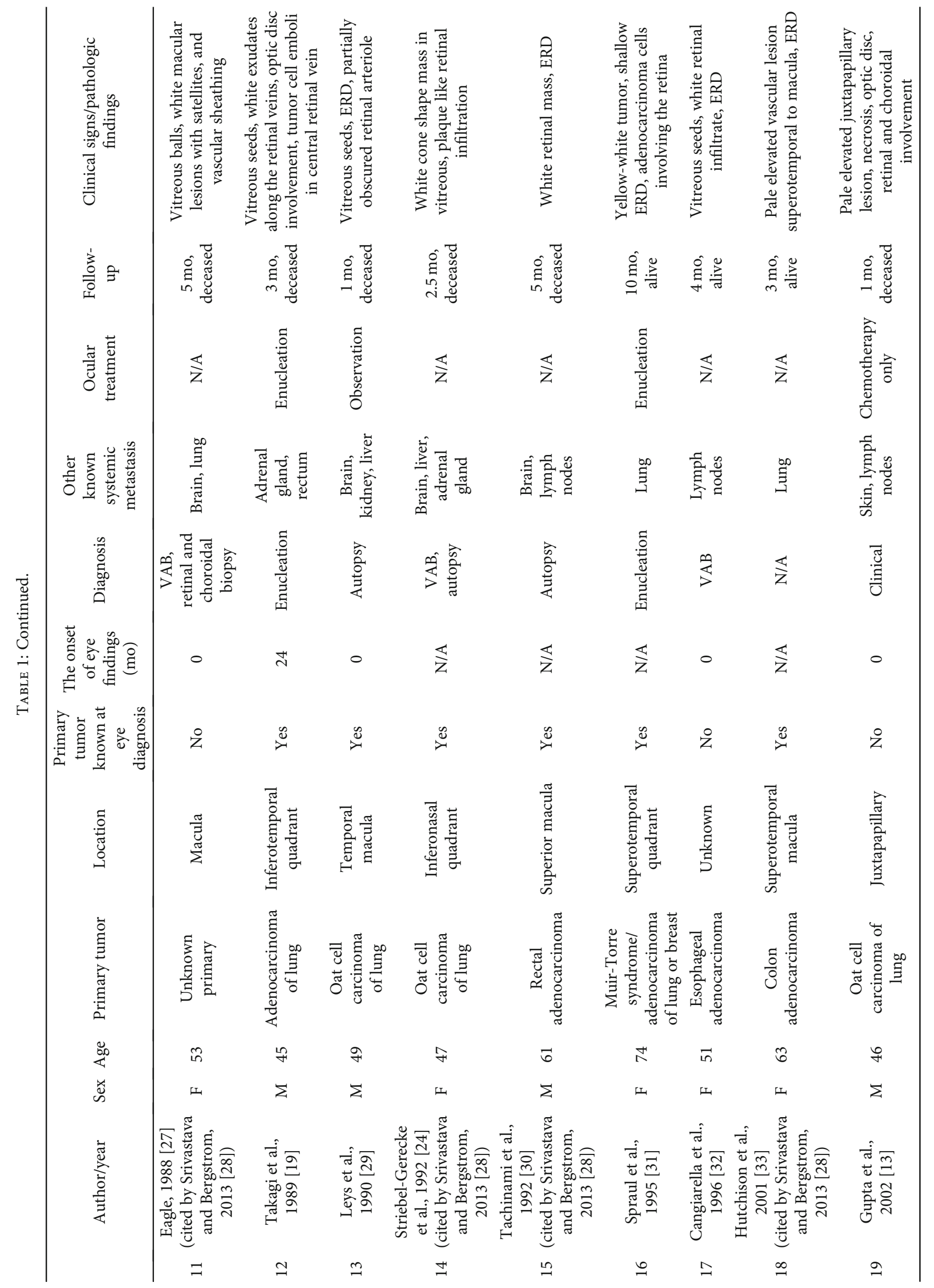




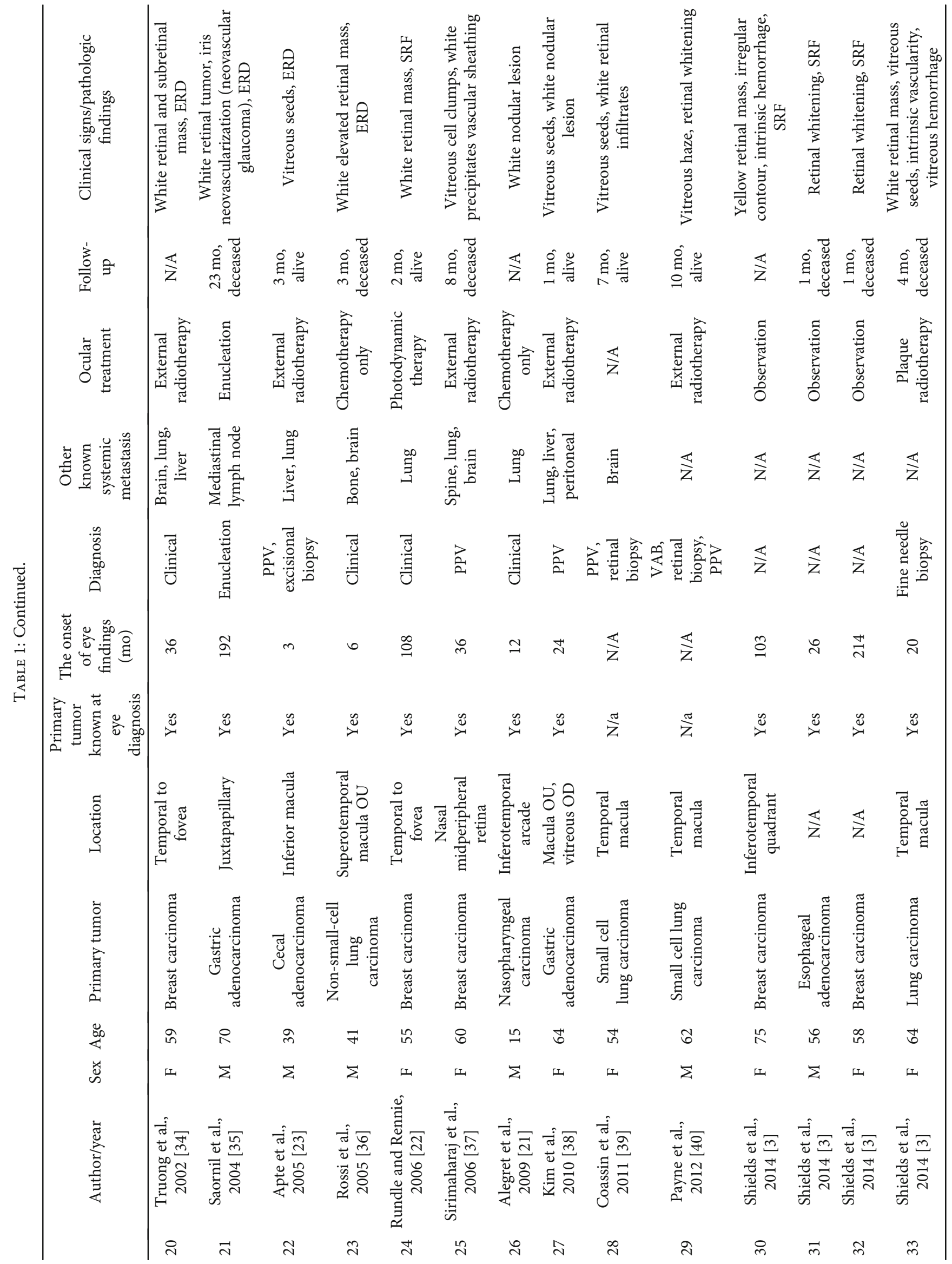




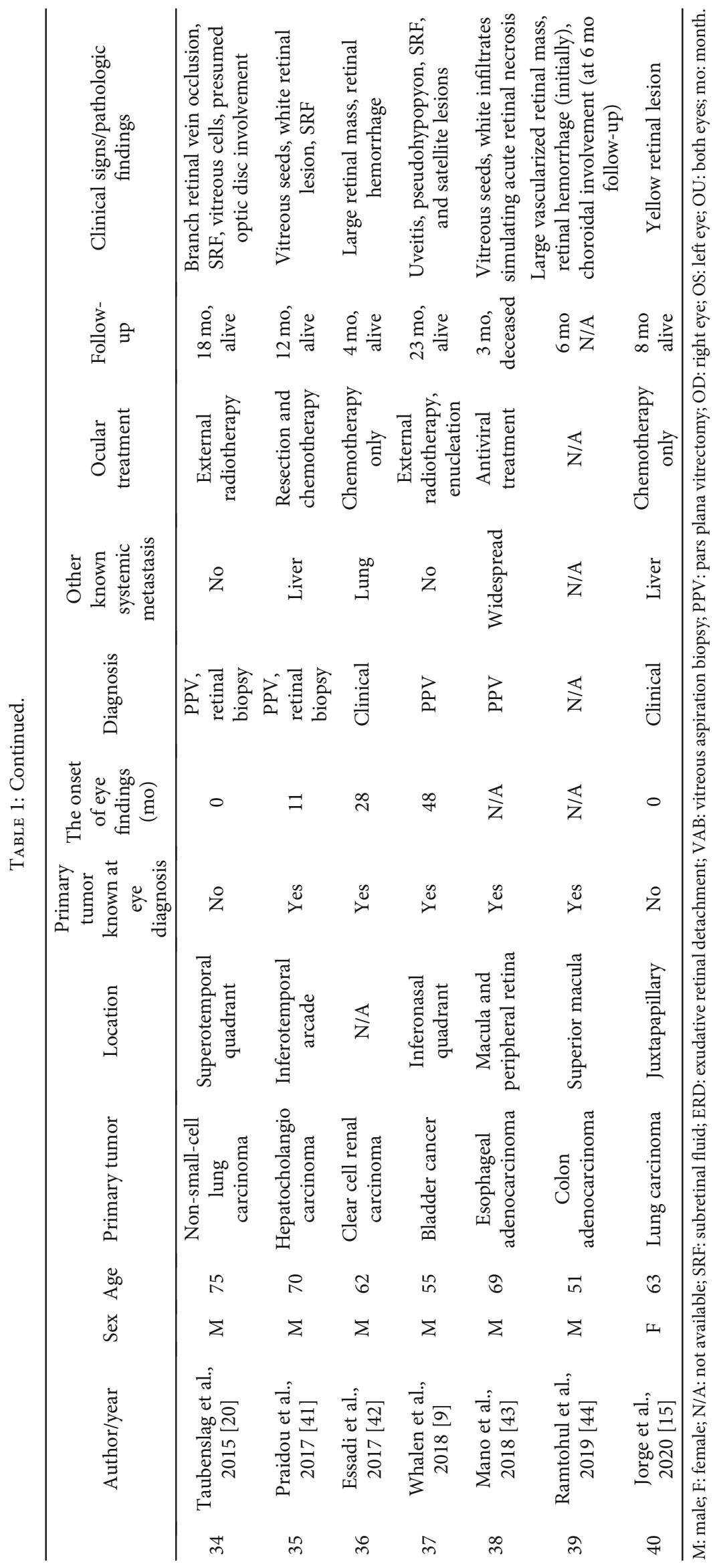


metastasis to the retina" or "metastatic carcinoma to the retina". The reference lists of the index publications identified through PubMed were also evaluated and relevant papers were retrieved. Chapters on metastatic tumors to the retina in major ophthalmology textbooks were reviewed. Articles written in non-English languages were also included. A total of 40 cases with carcinoma metastasis to the retina were found and reviewed for this study.

\section{Conflicts of Interest}

The authors declare that they have no conflicts of interest.

\section{References}

[1] B. Eliassi-Rad, D. Albert, and W. Green, "Frequency of ocular metastases in patients dying of cancer in eye bank populations," The British Journal of Ophthalmology, vol. 80, no. 2, pp. 125-128, 1996.

[2] A. K. O. Denniston and P. I. Murray, "Medical Retina," in Oxford American Handbook of Ophthalmology, pp. 454-455, New York, Oxford, 2nd edition, 2009.

[3] C. L. Shields, J. F. McMahon, H. T. Atalay, M. Hasanreisoglu, and J. A. Shields, "Retinal metastasis from systemic cancer in 8 cases," JAMA Ophthalmology, vol. 132, no. 11, pp. 1303-1308, 2014.

[4] W. Arap, M. Kolonin, M. Trepel, J. Lahdenranta, M. CardóVila, and R. Giordano, "Steps toward mapping the human vasculature by phage display," Nature Medicine, vol. 8, no. 2, pp. 121-127, 2002.

[5] K. Gündüz, J. A. Shields, C. L. Shields, and R. C. Eagle, "Cutaneous melanoma metastatic to the vitreous cavity," Ophthalmology, vol. 105, no. 4, pp. 600-605, 1998.

[6] K. Ramaesh, J. W. V. Marshall, S. B. Wharton, and B. Dhillon, "Intraocular metastases of cutaneous malignant melanoma : a case report and review of the literature," Eye, vol. 13, no. 2, pp. 247-250, 1999.

[7] P. H. Tang, L. Vajzovic, and P. Mruthyunjaya, "Retinal Metastatic Tumors," in Clinical Ophthalmic Oncology Retinal Tumors, A. D. Singh and B. E. Damato, Eds., pp. 101-113, Springer, Cleveland, 3rd edition, 2019.

[8] R. Klein, D. H. Nicholson, and M. N. Luxenberg, "Retinal metastasis from squamous cell carcinoma of the lung," American Journal of Ophthalmology, vol. 83, no. 3, pp. 358-361, 1977.

[9] K. E. Whalen, R. C. Eagle, and T. R. Vrabec, "Case of metastatic urothelial carcinoma of the retina and vitreous," Retinal Cases \& Brief Reports, vol. 12, no. 3, pp. 177-180, 2018.

[10] R. P. Koenig, D. L. Johnson, and R. H. Monahan, "Bronchogenic carcinoma with metastases to the retina," American Journal of Ophthalmology, vol. 56, no. 5, pp. 827-829, 1963.

[11] D. M. Robertson, C. P. Wilkinson, J. L. Murray, and D. D. Gordy, "Metastatic tumor to the retina and vitreous cavity from primary melanoma of the skin. Treatment with systemic and subconjunctival chemotherapy," Ophthalmology, vol. 88, pp. 1293-1301, 1981.

[12] U. Arisawa, "Über echtes karzinom in der retina," Klinische Monatsblätter für Augenheilkunde, vol. 17, pp. 386-399, 1914.

[13] M. Gupta, P. Puri, R. Jacques, and I. G. Rennie, "Retinochoroidal mass: a presenting feature of metastatic oat cell carcinoma of lung," European Journal of Ophthalmology, vol. 12, no. 6, pp. 550-552, 2002.

[14] R. M. Levy and G. VeneciaDe, "Trypsin digest study of retinal metastasis and tumor cell emboli," American Journal of Ophthalmology, vol. 70, pp. 778-782, 1967.

[15] D. M. Jorge, M. Labarrere, M. W. Rodrigues, C. L. Shields, and R. Jorge, "Simultaneous choroidal and retinal metastases from lung carcinoma," Retinal Cases \& Brief Reports, vol. 14, no. 1, pp. 90-95, 2020.

[16] J. R. Duke and F. B. Walsh, "Metastatic carcinoma to the retina," American Journal of Ophthalmology, vol. 47, no. 1, pp. 44-48, 1959.

[17] P. Piro, H. R. Pappas, Y. S. Erozan, R. G. Michels, and S. H. Sherman, "Diagnostic vitrectomy in metastatic breast carcinoma in the vitreous," Retina, vol. 2, no. 3, pp. 182-188, 1982.

[18] J. W. Smoleroff and S. A. Agatston, "Metastatic carcinoma of the retina," Archives of Ophthalmology, vol. 12, no. 3, pp. 359-365, 1934.

[19] T. Takagi, T. Yamaguchi, T. Mizoguchi, and T. Amemiya, "A case of metastatic optic nerve head and retinal carcinoma with vitreous seeds," Ophthalmologica, vol. 199, no. 2-3, pp. 123126, 1989.

[20] K. J. Taubenslag, S. J. Kim, A. Attia, and T. W. Abel, "Retinal metastasis from unknown primary : diagnosis, management and clinicopathologic correlation," Digital Journal of Ophthalmology, vol. 21, pp. 71-75, 2015.

[21] A. Alegret, C. M. Cebulla, S. R. Dubovy, L. Mutapcic, D. J. Hess, and T. G. Murray, "Pediatric nasopharyngeal carcinoma with retinal metastasis," Retinal Cases \& Brief Reports, vol. 3, no. 1, pp. 8-11, 2009.

[22] P. Rundle and I. Rennie, "Photodynamic therapy for solitary retinal metastasis from breast carcinoma," Eye, vol. 20, no. 12, pp. 1410-1412, 2006.

[23] R. S. Apte, C. Dibernardo, J. R. Pearlman et al., "Retinal metastasis presenting as a retinal hemorrhage in a patient with adenocarcinoma of the cecum," Archives of Ophthalmology, vol. 123, no. 6, pp. 850-853, 2005.

[24] S. U. Striebel-Gerecke, E. P. Messmer, and U. Landolt, "Retinale und vitreale metastase eines kleinzelligen bronchuskarzinoms," Klinische Monatsblätter für Augenheilkunde, vol. 200, no. 5, pp. 535-536, 1992.

[25] R. J. Flindall and K. O. Fleming, "Metastatic tumour of the retina," Canadian Journal of Ophthalmology, vol. 2, no. 2, pp. 130-132, 1967.

[26] S. E. Young, M. Cruciger, and J. Lukeman, "Metastatic carcinoma to the retina: case report," Ophthalmology, vol. 86, no. 7, pp. 1350-1354, 1979.

[27] R. C. Eagle Jr., Carcinomatous Retinitis, Presentation to the Eastern Ophthalmic Pathology Society, Hilton Head, SC, 1988.

[28] S. K. Srivastava and C. Bergstrom, "Retinal Metastases," in Retina, S. Ryan, C. Wilkinson, A. Schachat, D. Hinton, and C. Wilkinson, Eds., pp. 2254-2265, Saunders, Los Angeles, 5th edition, 2013.

[29] A. M. Leys, L. M. EyckVan, B. J. Nuttin, P. A. Pauwels, J. M. Delabie, and J. A. Libert, "Metastatic carcinoma to the retina," Archives of Ophthalmology, vol. 108, no. 10, pp. 1448-1452, 1990.

[30] K. Tachinami, T. Katayama, and N. Takeda, "A case of metastatic carcinoma to the retina," Nippon Ganka Gakkai Zasshi, vol. 96, no. 10, pp. 1336-1340, 1992. 
[31] C. W. Spraul, G. E. Lang, H. E. Grossnikiaus, and G. K. Lang, "Metastatic adenocarcinoma to the retina in a patient with Muir-Torre syndrome," American Journal of Ophthalmology, vol. 120, no. 2, pp. 248-250, 1995.

[32] J. F. Cangiarella, M. J. Suhrland, A. Cajigas et al., "Esophageal carcinoma metastatic to the retina. Diagnosis of a case by cytologic examination of intraocular vitreous washings," Acta Cytologica, vol. 40, no. 5, pp. 995-998, 1996.

[33] B. M. Hutchison, I. L. McAllister, and C. J. Barry, "Bowel carcinoma metastatic to the retina," Clinical \& Experimental Ophthalmology, vol. 29, no. 6, pp. 438-439, 2001.

[34] S. N. Truong, C. M. Fern, D. L. Costa, and R. F. Spaide, "Metastatic breast carcinoma to the retina: optical coherence tomography findings," Retina, vol. 22, no. 6, pp. 813-815, 2002.

[35] M. A. Saornil, G. Blanco, J. L. Sarasa, and G. Rabano, "Isolated metastasis of gastric adenocarcinoma to the retina : first presentation of systemic disease," Acta Ophthalmologica, vol. 82, no. 1, pp. 86-88, 2004.

[36] A. Rossi, A. Manto, P. Maione, and C. Gridelli, "Synchronous bilateral retinal metastases from lung adenocarcinoma," Tumori, vol. 91, no. 3, pp. 287-289, 2005.

[37] M. Sirimaharaj, A. P. Hunyor, and W. C. Chan, "Unusual ocular metastasis from breast cancer," Clinical \& Experimental Ophthalmology, vol. 6, no. 34, pp. 74-76, 2005.

[38] C. Y. Kim, C. W. Ha, and S. C. Lee, "Vitreous and retinal metastasis from gastric cancer," European Journal of Ophthalmology, vol. 20, no. 3, pp. 615-617, 2010.

[39] M. Coassin, K. B. Ebrahimi, and J. M. O’Brien, “Optical coherence tomography for retinal metastasis with unknown primary tumor," Ophthalmic Surgery, Lasers \& Imaging Retina, vol. 42, pp. 110-113, 2011.

[40] J. F. Payne, H. T. Rahman, and H. E. Grossniklaus, "Retinal metastasis simulating cytomegalovirus retinitis," Ophthalmic Surgery, Lasers \& Imaging, vol. 43, pp. 90-93, 2012.

[41] A. Praidou, S. Jacob, L. Irion, R. Sivaraj, C. Groenewald, and S. E. Coupland, "Retinal and vitreous metastases from hepatocholangiocarcinoma," BMC Cancer, vol. 17, no. 1, p. 430, 2017.

[42] I. Essadi, I. Lalya, M. Kriet, A. OmraniEl, and R. Belbaraka, "Successful management of retinal metastasis from renal cancer with everolimus in a monophthalmic patient : a case report," Journal of Medical Case Reports, vol. 11, no. 1, pp. 340-344, 2017.

[43] F. Mano, S. A. Lobue, K. C. Chang, and T. Mano, "Multimodal imaging of retinal metastasis masquerading as an acute retinal necrosis," International Journal of Retina and Vitreous, vol. 4, no. 1, p. 43, 2018.

[44] P. Ramtohul, D. Denis, and A. Comet, "Natural course of a retinal metastasis from colon adenocarcinoma," Ophthalmology, vol. 126, no. 6, p. 840, 2019. 